ks. Pavlo Fitsay ${ }^{1}$

Pontificio Istituto Orientale, Roma

\title{
Il significato della metafora di cuore nella spiritualità di Teofane il Recluso (1815-1894)
}

Durante il XIX secolo la Chiesa Ortodossa Russa ha testimoniato una stupenda fioritura di vita spirituale. Uno dei più grandi autori spirituali di quel periodo è il Vescovo Teofane il Recluso (in russo, Zatvornik), conosciuto nel mondo come Georgij Govorov². Il vescovo Teofane il Recluso fu uno dei grandi scrittori russi del XIX secolo: la sua bibliografia contiene 466 titoli. Gli scritti di spiritualità e di mistica sono i più importanti fra i moltissimi compilati da Teofane durante la permanenza a Vishen. Fanno parte dei suoi scritti anche le numerose lettere che trattano diversi argomenti: vita spirituale, vita cristiana, argomenti di vita e di fede, penitenza, comunione e il suo significato nella vita dell'uomo, cammino della salvezza, preghiera e vigilanza ${ }^{3}$. La parte degli scritti di Teofane il Recluso che ci interessa di più è quella riguardante la morale e l'ascesi, cioè: Compendio della morale cristiana; La via della salvezza; Lettere ${ }^{4}$. Nel

17 Padre mgr lic. Pavlo Fitsay nasce nella citta ucraina di Uzhgorod il 28 Aprile dell'anno 1986. Nel 2003 inizia a frequentare il greco-cattolico seminario diocesi di Mukachevo e sei anni dopo viene a Roma per frequentare il Pontificio Istituto Orientale, dove in 2012 ottiene la licenza in Teologia Orientale. L'indirizzo per la corrispondenza: pavlofitsay86@gmail.com.

2 Cf. C. di Valamo, L'arte della preghiera, Torino 1980, p. 15.

3 Cf. S. Bolshakoff, Incontro con la spiritualità russa, Torino 1990, p. 157.

4 Cf. T. Špidlik, Teofane il Recluso. Lo spirito e il cuore, Milano 2003, pp. 19-21. 
articolo, usando la metodologia della teologia, dopo la breve presentazione della biografia voglio spiegare il significato della metafora di cuore nella spiritualita di Teofane il Recluso.

\section{Biografia}

Georgij nacque il 10 gennaio 1815 nel villaggio di Cernavsk, nella provincia centrale di Viatka, vicino ad Orel, da Basilio, parroco di campagna e rettore del villaggio, uomo di valore e di grande pietà, e dalla donna, che era profondamente religiosa. Nel 1823 il giovane entrò nella scuola clericale di Livnij e qui si distinse fra i migliori e dopo averla finita fu mandato a studiare nel seminario di Orel nel 1829. Quando Georgij uscì dal seminario nel 1837 con le più alte votazioni entrò all'Accademia Ecclesiastica di Kiev, dove si distinse non meno che a Orel ${ }^{5}$. La sua intenzione di farsi monaco non era in alcun modo di ostacolo a questi obiettivi poiché, nella Chiesa russa, essere monaco significa poter accedere alla gerarchia, ossia occupare posti elevati nella società e nella Chiesa. Il 15 febbraio 1841 riceve l'abito monastico e il nome di Teofane ${ }^{6}$. Nel 1841 diventa rettore della scuola ecclesiastica di Kiev e nel 1842 Teofane fu nominato professore di logica e psicologia nonché ispettore del seminario di Novgorod; tre anni dopo è professore di teologia morale all'Accademia Ecclesiastica di Pietroburgo. Quindi nel 1855, rettore del seminario di Olonec e, nel 1857, rettore dell'Accademia pietroburghese. Il $1^{\circ}$ giugno 1859 l'archemandrita Teofane fu consacrato vescovo nella Laura pietroburghese di Sant'Alessandro Nevskij e, nominato al seggio di Tambov, l'occupò per quattro anni e per tre anni resse in seguito l'importante diocesi di Vladimir 7 . Nel 1866 Teofane inviò al Santo Sinodo la richiesta di essere esonerato dall'incarico e di potersi trasferire nel monastero di Vishen. Questa volta il suo desiderio trovò ascolto ${ }^{8}$. Teofane, giunto

5 Cf. S. Bolshakoff, Incontro con la spiritualità russa, op. cit., p. 152.

6 Cf. T. Špidlik, Teofane il Recluso..., op. cit., p. 10.

Cf. S. Bolshakoff, Incontro con la spiritualità russa, op. cit., p. 154.

8 Cf. T. Špidlik, Teofane il Recluso..., op. cit., p. 13. 
a Vishen il 3 agosto 1866, visse per ventotto anni consecutivi fino alla sua morte nel monastero, situato nel folto della foresta, organizzato e abitato da cento monaci, molto adatto alla contemplazione. Nei giorni festivi di solito celebrava anche lui. Durante questi sei anni Teofane ricevette diverse persone venute a trovarlo e in principio svolse le sue mansioni di abate, ma nel giorno di Pasqua del 1872 si ritirò in solitudine 9 . Sui dodici anni circa che Teofane visse come recluso abbiamo scarse notizie, si dice che trascorse giorni e notti in preghiera, piangendo ore intere sulla debolezza dell'anima umana e sulla misericordia divina che ci viene sempre in aiuto. Spesso leggeva e cantava ad alta voce, si dedicava inoltre al lavoro manuale, occupandosi di meccanica, di falegnameria, di rilegatura di libri e dipingendo icone. Nel tempo libero scriveva dei trattati di Teologia e delle lettere ${ }^{10}$. Il vescovo Teofane il Recluso negli ultimi anni si era preparato a ricevere lo skhima, e in questo tempo comincia a cucire il suo abito dello skhima ${ }^{11}$. Morì il 6 gennaio 1894 ed è stato seppellito nella cattedrale di Kazan ${ }^{12}$.

\section{Il cuore - l'organo spirituale}

Padre Tomaš Špidlik dice, che la spiritualità di Teofane si caratterizza con una sola parola: cuore, con quel termine possiamo cominciare ad esporre la sua dottrina antropologica. Teofane scrive nel suo libro Che cosa è la vita spirituale: "Nel cuore si concentrano tutte le energie del corpo e dell'anima. Esso è il barometro della nostra vita"13. Il cuore è il barometro della nostra vita perché tutto ciò che succede in noi o fuori di noi impressiona il nostro cuore e vi lascia una impronta. Esso disegna una scala di sentimenti diversi, quali la nostra lingua non può

\footnotetext{
9 Cf. S. Bolshakoff, Incontro con la spiritualità russa, op. cit., p. 155.

10 Cf. T. Špidlik, Teofane il Recluso.., op. cit., p. 14.

11 Cf. G. Tertyschnikov, Svjatitel Feofan Zatvornik i ego uchenie o spasenii, Moskva 1999, p. 160.

12 Cf. I. Krutikov, Svjatitel Feofan - zatvornik i podvizhnik Vishenskoj pustini, Moskva 1899, p. 135.

13 I. Krutikov, Svjatitel Feofan..., op. cit., p. 52.
} 
descrivere secondo la loro intensità e le loro sfumature, perché è molto povera di espressioni. Questi sentimenti sono mescolati, ma emerge sempre un elemento positivo o negativo, quello è piacevole o spiacevole, quello mi piace o dispiace ${ }^{14}$.

Per Teofane il cuore ha funzione di sentire tutto ciò che tocca la nostra persona. Esso sempre sente lo stato dell'anima e del corpo, e anche le diverse impressioni che l'anima e il corpo ricevono da loro - dagli oggetti che stanno attorno e che incontriamo, dalla vita esterna. Tutto quello costringe l'uomo a scegliere ciò che è piacevole e a rifiutare ciò che è spiacevole. La salute e la malattia dell'uomo, la vitalità e la fiacchezza del corpo, la fortezza e la stanchezza, la veglia e il torpore, e anche tutto ciò che l'uomo vede, sente, palpa, odora, assaggia, ricorda e immagina, tutto ciò che può pensare e riflettere, tutto ciò che sta facendo e ha fatto, le persone e le situazione dalla vita esterna - tutto questo si riflette nel cuore e lo provoca piacevolmente o spiacevolmente ${ }^{15}$.

Nel cuore si tiene tutto il movimento della vita spirituale dell'uomo, le verità vi ricevono il loro sigillo, le buone disposizioni vi si radicano. L'importante lavoro del cuore è donare il gusto, di rendere amabile. Quando il cuore immagina il mondo spirituale e le sue cose, deve prima di tutto provare la dolcezza e comunicare il calore, se lo fa la nostra anima riceve una vita nuova ${ }^{16}$.

Nel Compendio morale Teofane scrive che il cuore è considerato come la radice della sostanza umana, il centro di tutte le forze umane. Quelle forze dello spirito, dell'anima, e anche delle forze animali e corporali. $\mathrm{E}$ così il cuore ha un significato straordinario e eccezionale nell'uomo e nella sua esistenza. Perché attraverso il cuore l'essere umano entra in contatto con tutto ciò che esiste. L'autore definisce la duplice funzione del cuore: essere il centro delle forze e servire da base al contatto stabilito con tutto ciò che esiste fuori di ognuno ${ }^{17}$.

\footnotetext{
14 Cf. T. Špidlik, Il cuore e lo Spirito. La dottrina spirituale di Teofane il Recluso, Città del Vaticano 2004, p. 48.

15 Cf. Teofano il Recluso, La vita spirituale. Lettere, trad. di Garzaniti M., Roma 1989, lettera 8, p. 35.

16 Cf. Feofan Zatvornik, Put' ko spaseniju, Moskva 1908, p. 238.

17 Cf. Feofan Zatvornik, Nachertanie christianskago nravoucenija, Moskva 1895, p. 306.
} 
Per Teofane il cuore non può "fermarsi" neanche per un minuto, deve sempre stare attento per poter vedere il pericolo, simile al barometro prima della tempesta. Tutte le impressioni producono nel cuore un sentimento particolare, la lingua non ha vocaboli per distinguere tali sentimenti. Si può esprimere i nostri sentimenti in termini generali come gradevole-sgradevole, piacere-dispiacere, gaio-triste, gioia-pena, contentezza-afflizione, pace-tumulto, tranquillità-collera, speranza-timore, simpatia-antipatia. Così si deve fare attenzione nel proprio cuore per trovarvi l'uno o l'altro di questi sentimenti ${ }^{18}$.

L'importanza del cuore nell'economia della vita umana non è solo di condurre sotto le impressioni e di insoddisfazione ma anche è di sostenere l'energia di tutte le forze dell'anima e del corpo. Bisogna prendere in considerazione il fatto che le cose che ci piacciono e che ci stanno a cuore vengono fatte senza nessuna esitazione. Allora, quando qualcosa non ci sta a cuore, le braccia cadono e le gambe non si muovono. Quando l'uomo incontra una cosa piacevole, ma che non piace al cuore, allora perché ci sia un'armonia fra i due, bisogna che il cuore vi trovi qualcosa di positivo. Così lo zelo, quale è la forza dinamica della volontà proviene dal cuore. Lo stesso è nel lavoro intellettuale - l'oggetto che ci sta a cuore, si verifica più velocemente, accuratamente e i pensieri si muovono da sé ${ }^{19}$.

Se abbiamo la relazione con qualcuno, con cui stiamo bene e felici, allora qua c'è un punto di contatto che Teofane chiama sochustvie - la partecipazione con Dio. Se il cuore sta sempre nella dolcezza del peccato, dentro di esso c'è qualche cosa che sostituisce Dio. Perché il cuore è come sosud o l'anfora, se vi è Dio dentro, allora l'uomo ha la relazione con Lui e con il mondo esteriore ${ }^{20}$.

Il cuore ha una forma di conoscenza, ma una conoscenza molto diversa. Si parla di una conoscenza del tutto personale, il cui fine è molto particolare. L'esercizio dell'intelletto è basato sulla distinzione tra il vero

18 Cf. Teofano il Recluso, La vita spirituale. Lettere, op. cit., lettera 11, pp. 35-36.

19 Teofano il Recluso, La vita spirituale. Lettere, op. cit., lettera 11, p. 36.

20 Cf. F. Krjukov, Feofan Zatvornik. Pravoslavie i nauka. Rukovodstvennaja kniga izrecheij i pouchenij, Moskva 2009, p. 432. 
e il falso. Il giudizio separa questi due punti con esattezza ma anche con una freddezza impersonale. Il lavoro del cuore umano è stabilire parimenti una distinzione riguardo alla realtà, approva o disapprova. Ma non è facile esprimere il suo giudizio in parole chiare e precise, perché la distinzione che opera è tutta personale, poggia su ciò che per noi è buono o cattivo. Quando si apprezza la realtà in relazione a noi che si manifesta attraverso una moltitudine di impressioni ${ }^{21}$.

Teofane attesta che il cuore è la radice e il centro della vita. Esso è sempre pronto a giudicare lo stato umano, se è positivo o negativo, spingendo all'azione le altre forze e vigilando sulla loro attività. Possiamo affermare che il compito naturale del cuore è guidare la vita umana, ciò richiede di vigilare sullo stato del cuore e verificare tutti i sentimenti se sono buoni o cattivi. Perché l'uomo ha il dovere di tenere il cuore nelle mani, esporre e controllare i sentimenti, i gusti e le inclinazioni ${ }^{22}$.

\section{Azione del cuore - i sentimenti}

Teofane ha tratto dalla psicologia empirica del XIX secolo la sua divisione tripartita (intelligenza, volontà e sensibilità), per la quale il cuore e i "sentimenti" sono strettamente legati e anche egli è stato ispirato da Pseudo-Macario con la sua grazia compresa che si manifesta come un fuoco che scende nel cuore. Perché Teofane identifica con la massima semplicità la vita spirituale e la vita dei sentimenti ${ }^{23}$. Nel cuore e nella sua attività si riflettono tutte le forze dell'essere umano. L'autore divide i tre tipi del sentimento: i sentimenti spirituali, i sentimenti dell'anima e del corpo ${ }^{24}$, e anche li chiama "più bassi e grossolani, simili a quelli dell'animale"25.

${ }^{21}$ Cf. T. Špidlik, Il cuore e lo Spirito. La dottrina spirituale di Teofane il Recluso, op. cit., p. 48.

22 Cf. Feofan Zatvornik, Chto est' duhovnaja zhizn' i kak na nee nastroitsja, izdatel'stvo Zizn' s Bogom, Bruxelles 1996, lettera 10, pp. 26-27.

${ }_{23}$ Cf. T. Špidlik, Il cuore e lo Spirito. La dottrina spirituale di Teofane il Recluso, op. cit., p. 69-70.

24 Cf. F. Krjukov, Feofan Zatvornik. Pravoslavie i nauka..., op. cit., p. 436.

25 Cf. Feofan Zatvornik, Nachertanie christianskago nravoucenija, Moskva 1895, p. 322. 
Il cuore è il posto dei sentimenti spirituali, anche Teofane li chiama sentimenti religiosi, come aggregato di sentimenti spirituali. Essi si presentano nel cuore dopo la contemplazione del mondo spirituale. Per tutti gli uomini la fede è la fonte dei sentimenti, se l'uomo vive con la fede, nel cuore si versano i sentimenti spirituali come il timore di Dio, la riverenza, la devozione alla volontà di Dio, la speranza e l'amore. Ma il peccatore non ha quei sentimenti, perché senza la fede che è la loro fonte essi rimangono sul piano dei pensieri e dell'immaginazione. I sentimenti spirituali danno all'uomo senso di colpa davanti a Dio, vergogna di fronte a Lui, il pentimento, lo zelo di Dio, il sentimento del perdono in Gesù Cristo. Ma tutto questo è presente solo nell'uomo che ha fatto la conversione e la prassi spirituale ${ }^{26}$.

Teofane continua il suo pensiero dicendo che il peccatore non ha nessun sentimento religioso, ma quel sentimento riecheggia la paura e l'inquietudine. Così il peccatore ha paura di guardare verso Dio per cui può solo camminare nel buio del peccato, che è il risultato della sua vita perversa. L'uomo che pratica la vita spirituale si sente figlio di Dio, il quale diventa per lui il punto di ammirazione, per cui tutta la sua vita è nell'armonia ${ }^{27}$.

Non solo $i$ sentimenti spirituali s'impongono e si manifestano come i sentimenti del cuore, ma anche l'anima ha le sue esigenze naturali. Teofane afferma che i sentimenti dell'anima sono i movimenti del cuore che sorgono in essa come conseguenza dei cambiamenti che si producono nell'anima per la propria operazione. Essi si distinguono in sentimenti teorici, pratici ed estetici, perché provengono dall'intelletto e dalla volontà, o se siano una conseguenza della riflessione del cuore su se stesso ${ }^{28}$.

I sentimenti teorici nascono dalle relazioni del cuore che hanno lo scopo di conoscere la verità. Qua la ragione, con la necessità di conoscere, stimola al lavoro, e il frutto di questa conoscenza viene messo nel cuore. Il primo sentimento teorico è l'amore del sapere, e l'ultimo è la certezza di possedere la verità. Ma soltanto l'uomo con il cuore puro

26 Feofan Zatvornik, Nachertanie christianskago nravoucenija, op. cit., pp. 313-314.

27 Feofan Zatvornik, Nachertanie christianskago nravoucenija, op. cit., p. 315.

28 Cf. T. Špidlik, Il cuore e lo Spirito. La dottrina spirituale di Teofane il Recluso, op. cit., p. 74. 
può conoscere la verità e l'ordine delle cose, per cui il peccatore, stando nella vanità, nella frivolezza, nella disarmonia, non ha la possibilità di farlo ${ }^{29}$.

Il secondo tipo dei sentimenti presentato da Teofane è quello dei sentimenti pratici. Essi nascono nel cuore dopo l'incontro con le cose sottili o meravigliose. Il cuore si gode dal soggetto, perché è bello e meraviglioso e non dipende dai nostri interessi personali. La forza che sta sul fondamento di questi sentimenti si chiama gusto. Ci sono due gusti - il vero e lo sbagliato, e anche qua tutto dipende dallo stato dell'uomo. Così, l'uomo con il cuore puro, godendo le cose belle che mostrano il mondo spirituale e morale, può arrivare a conoscere il mondo divino. Questo gusto è la memoria del Paradiso e l'anticipazione del regno futuro. E al contrario, il gusto perverso e peccaminoso vuole soltanto le passioni che portano alla lussuria. Il peccatore non può vedere il bello nel divino, perché ha il gusto perverso ${ }^{30}$.

Il cuore come l'organo della vita spirituale è anche un organo corporeo, per cui la vita nella carne fa naturalmente parte della sua vita. Allora tranne $i$ sentimenti dell'anima, abbiamo i sentimenti del corpo che Teofane chiama "più bassi e grossolani, simili a quelli dell'animale" ${ }^{31}$. Quei sentimenti sono l'agitazione e le affezioni brusche del cuore, Teofane li chiama in latino affectus. Essi appartengono ai sentimenti di un livello inferiore, sfociano nella spontaneità dell'intelletto e della volontà e accompagnano gli accidenti particolari che il corpo subisce ${ }^{32}$.

I sentimenti come la sorpresa, lo stupore, la disattenzione, lo spavento, inibiscono la chiarezza della mente e la volontà viene inibita dalla paura e dalla rabbia. Tutti questi sentimenti torturano il cuore, che grazie ad essi passa da uno stato di gioia a quello della noia, si ribella e si abbandona all'invidia, è pieno di speranza o abbandonato alla disperazione ${ }^{33}$.

${ }^{29}$ Cf. Feofan Zatvornik, Nachertanie christianskago nravoucenija, Moskva 1895, pp. $318-320$.

30 Feofan Zatvornik, Nachertanie christianskago nravoucenija, op. cit., pp. 320-322.

31 Cf. T. Špidlik, Il cuore e lo Spirito. La dottrina spirituale di Teofane il Recluso, op. cit., p. 77.

32 Cf. Feofan Zatvornik, Nachertanie christianskago nravoucenija, op. cit., p. 322.

33 Feofan Zatvornik, Nachertanie christianskago nravoucenija, op. cit., pp. 322-323. 
Perché nello stato di peccato gli alti sentimenti spirituali sono indeboliti, e quelli "bassi" si ribellano con grande forza nell'uomo. L'uomo ha perso il controllo su se stesso e non ha l'armonia né dentro, né fuori di sés ${ }^{34}$.

\section{I sentimenti e la ragione}

Esponendo la dottrina del cuore nelle altre religioni e nelle tradizioni, abbiamo visto che il cuore viene presentato come il luogo dell'amore e della ragione. Da questo si può dedurre che l'amore e la conoscenza si uniscono nella persona umana e collaborano armoniosamente. L'intelletto è come "bilancia" che pesa oggettivamente il vero e il falso, è capace in sé di riconoscere la verità come verità, anche quando il cuore è depravato ${ }^{35}$.

Teofane il Recluso è lontano dal relativismo e dal soggettivismo gnoseologico. Ma in pratica il cuore ha un ruolo fondamentale nel buon funzionamento dell'intelletto, spinge la "bilancia" al contrario, ad entrare in azione. Il peccato falsa il giudizio, il cuore mai, perché esso rispetta la sovranità dei principi della logica. Così l'uomo è capace di conoscere ogni verità, e la via che conduce alla verità ultima passa attraverso la conoscenza di certe verità. Qui vediamo una conclusione importante che la disposizione del cuore influenza i nostri pensieri. L'intelletto non ha perso la sua capacità naturale di discernere il vero dal falso, ma l'essere nel peccato altera il giudizio del cuore ${ }^{36}$.

Allora il cuore puro è come un capo che dà ordini al suo subordinato - l'intelletto, così la stabilità del cuore contribuisce enormemente alla stabilità dei giudizi. Perché Teofane parla di questo procedimento che consiste nel ricondurre l'intelletto nel cuore, poiché se no, il minimo pensiero resta sfuggente ed instabile. La permanenza dentro di sé

34 Feofan Zatvornik, Nachertanie christianskago nravoucenija, op. cit., p. 323.

35 Cf. T. Špidlik, Il cuore e lo Spirito. La dottrina spirituale di Teofane il Recluso, op. cit., p. 82.

36 T. Špidlik, Il cuore e lo Spirito. La dottrina spirituale di Teofane il Recluso, op. cit., p. 83. 
apre il mondo spirituale che è la condizione per riscaldare i pensieri spirituali ${ }^{37}$.

Teofane ritorna sul punto di inserire la conoscenza razionale nel tutto della persona umana, per lui è importante mettere i pensieri nel cuore, unire la testa al petto, i giudizi ai sentimenti. Quando c'è qualche domanda non si può filosofare e fare le nuove domande a se stesso, ma bisogna mettere l'oggetto dell'osservazione nel proprio cuore ${ }^{38}$. Si deve vivere nel cuore, ma non nella testa, e solo se c'è il bisogno della vita spirituale si può lavorare con la ragione, la quale deve essere collocata nel cuore $^{39}$. Per esempio, quando c'è il problema dell'incredulità ciò non è il problema della ragione, ma è il problema del cuore corrotto. La colpa della ragione consiste nella sua obbedienza al cuore corrotto, il quale non pensa alla verità oggettiva, ma obbedisce alla volontà del cuore impuro. E gli argomenti forti della verità per la ragione sembrano deboli, per cui la ragione rimane confusa e accecata ${ }^{40}$.

Con la ragione possiamo conoscere soltanto una parte della verità, una specie di proiezione di quest'ultima e per poter conoscere tutta la verità nella sua oggettivita si ha bisogno della convinzione sincera (in russo serdechnoe ubeždenie). La vera conoscenza del tutto si dà solo nello Spirito. Il cuore che serve Dio ed è pulito per lo Spirito Santo diventa lo stato della tabula rasa, dove lo Spirito può scrivere il segreto della conoscenza di Dio ${ }^{41}$.

Infine Teofane dice che la conoscenza di Dio è nel rifiuto della ragione. Se tu rifiuti la tua ragione, diventerai una vera intelligenza. Possiamo affermare insieme al nostro autore che la vera conoscenza di Dio sta più nel sentire che nel capire ${ }^{42}$.

37 Cf. Feofan Zatvornik, Put' ko spaseniju, Moskva 1908, p. 220.

38 Cf. Feofan Zatvornik, Turning the heart to God, trad. di I. Zhiltsov, K. Kaisch, California 2001, p. 65.

${ }_{39}$ Cf. Feofan Zatvornik, Pis'ma k raznim licam o raznih predmetah viri I zhizni, Moskva 1892 , lettera 44, p. 331.

40 Cf. Feofan Zatvornik, Kratkie misli na kazhdij denj goda po cerkovnomu chteniju iz Slova Bozhija, Uspenskij Pskovo-Pecherskij monastir 1991, p. 73.

${ }_{41}$ Cf. F. Krjukov, Feofan Zatvornik. Pravoslavie i nauka..., op. cit., p. 236.

${ }^{42}$ Cf. Feofan Zatvornik, Kratkie misli na kazhdij denj goda..., op. cit., p. 274. 


\section{Il cuore e la volontà}

La libera volontà si esercita attraverso una scelta tra il bene e il male, e poiché, secondo la Scrittura, questa scelta è fatta dal cuore, lo schema volontarista identifica il cuore con le decisioni. Al campo dei sentimenti, l'anima si contenta di subire e di provare, mentre il cuore reagisce e lascia intravedere l'intervento della volontà. Così il cuore prende anche decisioni e delle responsabilità. Per Teofane la volontà e il cuore sono due facoltà distinte, la loro collaborazione è indispensabile. La conoscenza deve discendere dalla testa al cuore, e per essere veramente virtuosa l'azione della volontà deve provenire dal cuore ${ }^{43}$.

Le scelte della volontà sono seguite da operare perché la volontà è una forza operativa e il suo bisogno essenziale è quello di vivere e operare ${ }^{44}$. Costruire la nostra vita sulla terra apparterrà alla volontà - intraprendere azioni, piani, disposizioni, comportamento, è una possibilità dell'aspirazione e della posizione, allora tutto questo l'uomo esprime dentro di sé. Il punto di vista della volontà è il bene. I tipi dell'azione della volontà sono il desiderio e l'avversione, il segreto della vita è desiderare il bene e avversare il male ${ }^{45}$.

La fonte o l'inevitabilità dei desideri è l'incompletezza della nostra esistenza. Il sentimento della incompletezza costringe l'uomo a cercare le cose per complementarsi. Il soggetto della ricerca dell'uomo è il bene, così il massimo bene è Dio ${ }^{46}$. L'atto umano è sempre ordinato dalla libera volontà. Questa esige innanzitutto che le siano proposti i motivi per agire, come un bisogno, una utilità, una soddisfazione e un valore. Ed è il cuore che deve dettare questi motivi, perché è esso che desidera il bene, così l'atto umano è comandato dalla libera volontà che risiede nel cuore. Se la sua volontà è libera, l'uomo è responsabile dei suoi atti o sono beni o mali ${ }^{47}$.

pp. 97-99.

${ }^{43}$ Cf. T. Špidlik, Il cuore e lo Spirito. La dottrina spirituale di Teofane il Recluso, op. cit.,

${ }^{44}$ Cf. T. Špidlik, Teofane il Recluso..., op. cit., p. 142.

45 Cf. Feofan Zatvornik, Nachertanie christianskago nravoucenija, op. cit., p. 279.

46 Feofan Zatvornik, Nachertanie christianskago nravoucenija, op. cit., p. 280.

${ }_{47}$ Cf. T. Špidlik, Il cuore e lo Spirito. La dottrina spirituale di Teofane il Recluso, op. cit., p. 99. 
La partenza della volontà è lo zelo o il desiderio. Le cause che lo producono sono il piacere, l'utilità e la necessità. Lo zelo molto importante per queste forze operative poiché senza di esso le forze, rilassandosi, perdono la vigilanza. Nel momento della loro presenza il desiderio suscita e accende lo zelo. Se una volta abbiamo soddisfatto, in qualche modo, i nostri bisogni, al nascere del desiderio primario, nasce anche il desiderio di quella cosa con la quale abbiamo soddisfatto il bisogno fondamentale. Così un desiderio si riferisce sempre a un determinato oggetto che soddisfa il bisogno. Se qualche bisogno è stato soddisfatto in maniera diversa - di conseguenza, quando nasce di nuovo lo stesso bisogno, nascono anche diversi desideri verso l'una o l'altra cosa che possa soddisfare quel bisogno. E soltanto i desideri i quali vogliono essere soddisfatti nascono nel cuore, e i bisogni che stanno in fondo dei desideri non si vedono ${ }^{48}$.

Come abbiamo già detto il soggetto della volontà è Dio, che è quello dell'uomo con il puro cuore. La volontà dell'uomo peccatore è guidata a lui stesso, così l'uomo è diventato soggetto del desiderio e altre cose sono i mezzi, perché ama se stesso. Così vediamo che il posto importante nell'anima dell'uomo occupa l'egoismo. E da quel peccato si comincia a determinare tutto nell'uomo, la volontà comincia già a chiamare il male come il bene, i desideri sono depravati e non servono per il bene dell'uomo, la ragione sta nell'ignoranza, la carne vuole solo il piacere e da qui l'uomo ha perso l'armonia con tutti e con se stesso ${ }^{49}$.

Per educare la volontà si deve formare in essa la virtù come l'umiltà, la conformità, la compiacenza che devono fondersi con la volontà e in questo modo possono diventare la natura di essa, e potranno controllare le nostre operazioni. Tutto parte dal timore di Dio, dopo passa alla virtù e alla fine si fa più forte con l'amore ${ }^{50}$.

${ }^{48}$ Cf. T. Špidlik, Teofane il Recluso..., op. cit., p. 142.

49 Cf. Feofan Zatvornik, Nachertanie christianskago nravoucenija, op. cit., pp. 280-281.

50 Cf. Feofan Zatvornik, Put' ko spaseniju, Moskva 1908, p. 238. 


\section{Il cuore e lo spirito}

Cosa significa lo spirito? I filosofi greci parlavano del carattere immateriale e l'hanno identificato con l'anima che è per natura sua "spirituale”. Per i cristiani è andata sempre più affermandosi un'antropologia che parla dell'uomo come di un essere spirituale a motivo della presenza dello Spirito Santo in lui. Così il grande mistero della vita cristiana è il mistero delle molteplici relazioni dello spirito umano con lo Spirito di Dio ${ }^{51}$.

Teofane segue la tradizione della Bibbia che associa il cuore all'anima è allo spirito (ruah). Lo zelo e la collera sono fenomeni tanto dello spirito quanto del cuore. Nella parte delle sue accezioni morali e religiose, il cuore può essere sostituito dallo spirito, è sufficiente ricordare il cuore contrito e lo spirito contrito (Sal 51, 19), lo spirito nuovo e il cuore nuovo $(E z 36,26)$. Il cuore appartiene in proprio all'uomo, mentre spesso lo spirito è in rapporto stretto con Dio che lo dona e lo riprende ${ }^{52}$.

Allora che cosa è lo spirito? Possiamo dire che è la forza, che è stata soffiata da Dio nella faccia dell'uomo, e in questo sta la differenza tra l'uomo e il mondo. Lo spirito, visto come la forza che scende da Dio e può conoscere Dio, può cercarLo e solo in Lui troverà la pace ${ }^{53}$. Teofane dice che lo spirito è la forza che innalza l'uomo dal visibile all'invisibile, dal contingente all'eterno, dalla creatura al creatore, che caratterizza l'uomo e lo distingue da tutte le altre creature terrene. Questa forza può essere indebolita secondo i diversi gradi e le sue esigenze possono essere interpretate ed equivocate, ma non la si può soffocare o sradicare del tutto $^{54}$. E se per caso lo spirito dell'uomo cade e vive nel peccato, rimane sempre spirito e rifletterà qualcosa di celeste ma non in modo chiaro ${ }^{55}$.

L'autore continua dicendo che il cuore è la sede della grazia, il punto di contatto tra Dio e l'uomo. Questa unione si deve manifestare nell'attività principale del cuore, nello sbocciare dei sentimenti. Lo spirito ha le stesse esigenze e desideri del corpo e dell'anima che si congiungono, così

\footnotetext{
51 Cf. T. Špidlik, Teofane il Recluso..., op. cit., p. 61.

52 Cf. T. Špidlik, Il cuore e lo Spirito. La dottrina spirituale di Teofane il Recluso, op. cit., p. 102.

53 Cf. F. Krjukov, Feofan Zatvornik. Pravoslavie i nauka..., op. cit., p. 236.

54 Cf. Feofan Zatvornik, Chto est' duhovnaja zhizn'..., op. cit., lettera 11, p. 33.

55 Cf. Feofan Zatvornik, Slova na gospodskie, bogorodicnie i torzhestvennie dni, Moskva 1883,
} p. 200. 
l'uomo aspira con tutto il suo cuore al bene unico e supremo, cioè a Dio ${ }^{56}$. Lo spirito umano cerca sempre la verità che è Dio per mezzo del desiderio spirituale. Il cuore unisce il desiderio dello spirito alla tendenza dell'anima in un sentimento unico, potente nell'uomo in stato di grazia. L'anima e lo spirito sono stati creati per collaborare perché hanno un soggetto la verità, Dio $^{57}$.

Allora Dio è il soggetto della contemplazione dello spirito nel cuore, come il fondamentale bene per l'uomo. Perciò la pienezza della vita cristiana è la contemplazione di Dio, e quella attività appartiene allo spirito umano. Ma prima di tutto il cuore deve essere pulito e libero da tutte le cose che ostacolano lo spirito nella contemplazione ${ }^{58}$.

Nel commento al Salmo 118 Teofane parla dell'occhio spirituale che contempla il mondo divino e spirituale, questo è lo spirito, il quale è chiamato dal nostro autore "mente", in russo "um". Ma qua Teofane non parla della mente nel senso della ragione o dell'intelletto, che chiama vedeni $e^{59}$, ma nel senso dello spirito umano. Quello spirito contempla nel cuore Dio e confessa la Sua provvidenza, lo spirito che educa all'obbedienza a Lui, cerca di trovarLo e vederLo come il suo ultimo bene. Quell'occhio si apre soltanto con la grazia dello Spirito Santo ${ }^{60}$.

Lo spirito ha sul cuore un'influenza decisiva per il suo normale sviluppo. Esso soffia dove vuole, è prima di tutto un dono che dipende dalla libera decisione divina. Il dominio di questo dono dipende dalle nostre forze e la sua luce può essere intensificata o attenuata, o addirittura anche spenta a seconda che essa sia ricevuta in un cuore dove traboccano le passioni o al contrario in un cuore che riflette tutta la bellezza del cielo. Ma per vivere secondo lo spirito pienamente umano si deve

56 Cf. T. Špidlik, Il cuore e lo Spirito. La dottrina spirituale di Teofane il Recluso, op. cit., p. 102.

57 Cf. Feofan Zatvornik, Nachertanie christianskago nravoucenija, op. cit., pp. 316-317.

58 Cf. Feofan Zatvornik, Tolkovanie poslanija Sv. Apostola Pavla k Efesejam, Moskva 1893, pp. 245-246.

59 In russo "vedenie" o "vedat" significa la conoscenza o vedere. Talvolta Teofane usa il termine um come lo spirito umano o come la mente o la ragione. "La ragione - "vouc" corisponde completamente alla parola "lo spirito" - Cf. F. Krjukov F., Feofan Zatvornik. Pravoslavie i nauka..., op. cit., p. 334.

60 Cf. Feofan Zatvornik, Psalom Sto-Osmnadcatyj istolkovanyj, Moskva 1891, p. 383. 
purificare il cuore ${ }^{61}$. E così lo Spirito di Dio deve venire e raccogliere i pezzi schiacciati dello spirito umano, per formare nel cuore il nuovo uomo spirituale ${ }^{62}$.

\section{Conclusione}

Che cosa possiamo dire alla fine del nostro percorso? Prima di tutto vediamo che Teofane ha basato tutti i suoi pensieri sul cuore sulla tradizione patristica, specialmente nell'impiego della Filocalia, che egli ha tradotto in russo Dobrotoljubie. Abbiamo visto che Teofane in alcuni punti ha ereditato dai Padri la loro visione del cuore, ossia quella in cui essi paragonano e a volte identificano il cuore con la mente (um), per cui spesse volte questi due termini sono usati come sinonimi, per esempio Massimo il Confessore identifica la mente con il cuore, perché nella mente abita Cristo. Un'altra fonte dei pensieri sulla teologia del cuore era l'esempio della la vita spirituale di Tichon di Zadonsk, da cui scritti fu influenzato il nostro autore.

Usando la metafora di cuore per spiegare la vita interiore Teofano mette l'accento sul cuore come il nostro organo spirituale, che è il luogo in cui avviene l'incontro fra Dio e l'uomo e che porta la nostra preghiera verso Dio. Teofane vuole mostrare che la preghiera fa parte della nostra natura ed è un'elevazione verso Dio. Spiega che il cuore è una specie di motore di tutte le energie dell'anima e del corpo, ed è la fonte principale della vita spirituale e corporale. Solo nel cuore si può contemplare Dio, si può "vedere" con gli occhi spirituali e con il cuore puro. La seconda idea consiste nell'attenzione e nella custodia del cuore. Il cuore è molto prezioso, per cui i nostri nemici vogliono prenderlo e metterlo nell'errore. L'autore discerne tutte le azioni del nemico contro il cuore, spiega come si può respingere l'assalto del diavolo, e fa di noi il guerriero dell'esercito di Cristo.

${ }_{61}$ Cf. T. Špidlik, Il cuore e lo Spirito. La dottrina spirituale di Teofane il Recluso, op. cit., p. 108.

${ }_{62}$ Cf. Feofan Zatvornik, Slova na gospodskie, bogorodicnie i torzhestvennie dni, op. cit., p. 200. 


\section{Summary}

Il significato dell cuore nella spiritualita di Teofane il Recluso (1815-1894).

La spiritualita dell cuore di Teofane il Recluso nasce nella tradizione patristica Orientale e anche nella tradizione della Filocalia. Teofane cerca i sentimenti spirituali nella tradizione dei Padri Orientali come gli azioni del cuore come il ragione (in russo "um"). Nella teoria di Teofane il cuore come l'organo spirituale é il punto dell'incontro e relazione tra Dio e l'uomo. Il Cristiano puo "vedere" Dio solo con gli occhi sprituali e con il cuore puro. Teofane dice che il cuore é il posto dove si svolge la lotta spirituale contro le passioni.

Parole chiave: Teofane il Recluso, la spiritualita del cuore, I sentimenti spirituali

Słowa kluczowe: Teofan Pustelnik, duchowość serca, zmysły duchowe

\section{Abstract}

The meaning of the metaphor of the heart in the spirituality of Theophane the Recluse (1815-1894)

Spirituality Theophane the Recluse refers to the metaphor of the heart, the source of which is found in the patristic tradition, particularly in the Philokalia. Theophan drawing from the heritage of the Fathers of the Church, appealed especially to the theory of the spiritual senses and identify the heart with the mind (rus. "um"). Using the metaphor of the heart tried to explain the Christian spiritual life, the heart would be a place of a spiritual encounter between God and man. Human being can 'see' God only by a spiritual eyes and a pure heart. Theophan also drew attention to the spiritual gifts of discernment and vigilance in the heart.

Keywords: Theophane the Recluse, spirituality of the heart, spiritual senses

\section{Bibliografia}

Adnes P., Hésychasme, in: Dictionnaire de Spiritualité, V/7, Paris 1968.

Battaglia S., Il cuore, in: Grande dizionario della lingua italiana, V/3, Torino 1971. 
Behm J., Kardia, in: Grande lessico del Nuovo Testamento, a cura di F. Montagnini, G. Scarpat, O. Soffritti, V/5, Brescia 1969.

Bolshakoff S., Incontro con la spiritualità russa, Torino 1990.

Caritone di Valamo, L'arte della preghiera, Torino 1980.

Chevalier J., Gheerbrant A., Il cuore, in: Dizionario dei simboli, a cura di I. Sordi, V/1, Milano 1996.

Concetti L., Il cuore, in: Dizionario Interdisciplinare di scienza e fede, a cura di

G. Tanzella-Nitti, A. Strumia, V/1, Roma 2002.

De Fraine J., Vanhoye A., Il cuore, in: Dizionario di Teologia biblica, a cura di X. LeonDufour, Genova-Milano 2005.

Dobrotoljubie, V/3, Moskva 1895.

Feofan Zatvornik, Četire slova o molitve, Moskva 1911.

Feofan Zatvornik, Chto est' duhovnaja zhizn' i kak na nee nastroitsja, izdatel'stvo Zizn' s Bogom, Bruxelles 1996.

Feofan Zatvornik, Kratkie misli na kazhdij denj goda po cerkovnomu chteniju iz Slova Bozhija, Uspenskij Pskovo-Pecherskij monastir 1991.

Feofan Zatvornik, La vita spirituale, Lettere, trad. di M. Garzaniti, Roma 1989.

Feofan Zatvornik, Mysli na každij den' goda po cerkovnim čtenijam iz Slova Božija, Moskva 1881.

Feofan Zatvornik, Nachertanie christianskago nravoucenija, Moskva 1895.

Feofan Zatvornik, Pis'ma k raznim licam o raznih predmetah virii I zhizni, Moskva 1892.

Feofan Zatvornik, Pisjma o hristianskoj zhizni, Moskva 1908.

Feofan Zatvornik, Psalom Sto-Osmnadcatyj istolkovanyj, Moskva 1891.

Feofan Zatvornik, Put' ko spaseniju, Moskva 1908.

Feofan Zatvornik, Slova na gospodskie, bogorodicnie i torzhestvennie dni, Moskva 1883.

Feofan Zatvornik, Svjatie otci o molitve i trezvenii, Moskva 1997.

Feofan Zatvornik, Tolkovanie poslanija Sv. Apostola Pavla k Efesejam, Moskva 1893.

Feofan Zatvornik, Tolkovanie poslanija Sv. Apostola Pavla k Kolossaem i Filimonu, Moskva 1892.

Feofan Zatvornik, Turning the heart to God, la traduzione inglese di I. Zhiltsov, K. Kaisch, California 2001.

Garsìa de la fuente O., Il cuore, in: Enciclopedia della Bibbia, V/2, Torino 1969.

Hoffman P., Il cuore, in: Dizionario Teologico, a cura di G. Riva, V/1, Brescia 1966.

Krjukov F., Feofan Zatvornik. Pravoslavie i nauka. Rukovodstvennaja kniga izrecheij i pouchenij, Moskva 2009.

Krutikov I., Svjatitel Feofan-zatvorniki podvizhnik Vishenskoj pustini, Strasnoj buljvar, Moskva 1899.

La Filocalia. Testi di ascetica e mistica della Chiesa Orientale, a cura di G.P. Vannucci, Libreria Editrice Fiorentina, Firenze 1963.

Lemaire A., Il cuore, in:Dizionario Enciclopedico della Bibbia, a cura di R. Penna, Roma 1996.

Marchetti-Salvatori B., Il cuore, in: Dizionario Enciclopedico Spiritualità, a cura di E. Ancilli, V/1, Roma 1990. 
Meslin M., Il cuore, in: The Encyclopedia of Religion, a cura di M. Eliade, V/5-6, New York 1993.

Molinaro A., Il cuore, in: Enciclopedia filosofica, a cura di V. Melchiorre, V/3, Milano 2006.

Moschetti A., Il cuore, in: Enciclopedia filosofica, V/1, Istituto per la collaborazione culturale, Venezia-Roma 1957.

Mystic treatises by Isaac of Nineveh, Amsterdam 1923.

Parole del deserto. Detti inediti di Iperechio, Stefano di Tebe e Zosima, a cura di L. Cremaschi, Magnano 1992.

Pierre M., Lessico del deserto. Le parole della spiritualità, a cura di V. Lanzarini, Comunita di Bose 1998.

Poor M., Orthodox prayer life. The interior way, New York 2003.

Rocco U., La sobrietà, in: Dizionario enciclopedico di spiritualità, a cura di E. Ancilli, V/3, Roma 1990.

Sand A., Kardia, in: Dizionario esegetico del Nuovo Testamento, a cura di H. Balz, G. Schneider, Brescia 2004.

Špidlik T., Il cuore e lo Spirito. La dottrina spirituale di Teofane il Recluso, Città del Vaticano 2004.

Špidlik T., Il cuore nella spiritualità russa, in: Cuore del Cristo: cuore dell'nomo, Miscellanea III, Adria-Napoli 1982.

Špidlik T., L'arte di purificare il cuore, Roma 2008.

Špidlik T., La preghiera, in: Dizionario enciclopedico dell'Oriente Cristiano, a cura di E. Farrugia, Roma 2000.

Špidlik T., Lo spirito e il cuore, Milano 2003.

Špidlik T., Pregare nel cuore, Roma 2008.

Stolz F., Il cuore, in: Dizionario teologico dell'Antico Testamento, a cura di G. L. Prato, V/1, Torino 1978.

Tertyschnikov G., Svjatitel Feofan Zatvornik i ego uchenie o spasenii, Moskva 1999.

Vlakhos I., Pravoslavnaja psihoterapija, Moskva 2004.

Vosplamenenie uma $v$ duhovnoj pustine. Isaak Sirin, a cura di A. Muravjov, Moskva 2008.

Vyačeslavcev B., Serdce v hristianskoj i indijskoj mistike, Paris 1929.

Zadonskij T., Sokrovische duhonvoe ot mira sobiraemoe, V/3, Moskva 2003. 